\title{
Perpendicular magnetic anisotropy in chemically disordered FePd-FeV(100) alloy thin films
}

\author{
C. Clavero, ${ }^{\text {a) }}$ J. M. García-Martín, G. Armelles, and A. Cebollada \\ Instituto de Microelectrónica de Madrid-IMM (CNM-CSIC), Isaac Newton 8-PTM, E-28760 Tres Cantos, \\ Madrid, Spain \\ Y. Huttel \\ Instituto de Ciencia de Materiales de Madrid (ICMM-CSIC), E-28049 Cantoblanco, Madrid, Spain
}

S. Estradé, J. Arbiol, and F. Peiró

EME Electronic Materials and Engineering, Department Electronics, University of Barcelona, Martí i Franquès 1, E-08028 Barcelona, Spain

\author{
LI. Balcells \\ Instituto de Ciencia de Materiales de Barcelona-ICMAB (CSIC), Campus de la Universidad Autònoma \\ de Barcelona, E-08193 Bellaterra, Spain
}

(Received 3 October 2005; accepted 9 February 2006; published online 10 April 2006)

\begin{abstract}
We find that the use of $\mathrm{V}(100)$ buffer layers on $\mathrm{MgO}(001)$ substrates for the epitaxy of FePd binary alloys yields to the formation at intermediate and high deposition temperatures of a FePd-FeV mixed phase due to strong $\mathrm{V}$ diffusion accompanied by a loss of layer continuity and strong increase of its mosaic spread. Contrary to what is usually found in this kind of systems, these mixed phase structures exhibit perpendicular magnetic anisotropy (PMA) which is not correlated with the presence of chemical order, almost totally absent in all the fabricated structures, even at deposition temperatures where it is usually obtained with other buffer layers. Thus the observed PMA can be ascribed to the $\mathrm{V}$ interdiffusion and the formation of a FeV alloy, being the global sample saturation magnetization also reduced. (C) 2006 American Institute of Physics. [DOI: 10.1063/1.2187413]
\end{abstract}

\section{INTRODUCTION}

The fabrication of binary FePt and FePd alloys is of great fundamental and technological interests. These alloys in their chemically ordered phase $\left(L 1_{0}\right)$ exhibit large perpendicular magnetic anisotropy (PMA): $K_{u} \sim 10^{7} \mathrm{~J} / \mathrm{m}^{3}$ in $\mathrm{FePt}$ and $K_{u} \sim 9 \times 10^{5} \mathrm{~J} / \mathrm{m}^{3}$ in FePd. ${ }^{1}$ Chemical order is characterized by the alternation of pure $\mathrm{Fe}$ and $\mathrm{Pd}(\mathrm{Pt})$ atomic planes along a cubic (001) direction, which as a consequence suffers a tetragonal distortion with respect to the cubic lattice, and becomes the axis for easy magnetization. Therefore, chemically ordered $L 1_{0}$ films grown along the (001) direction exhibit PMA. On the other hand, in magnetic nanoparticle systems the thermal stability of the magnetization in individual nanoparticles scales with the anisotropy constant $\left(K_{u}\right)$ and the nanoparticle volume; ${ }^{2}$ therefore with the presence of such a high magnetic anisotropy phase it is possible to reduce strongly the nanoparticle size while avoiding the so called superparamagnetic limit. These features make them excellent candidates to develop ultrahigh density magnetic media with naturally separated and stable bits. ${ }^{3,4}$ The onset of chemical order and therefore large magnetic anisotropy in FePd and FePt binary alloys is associated with the chemical order degree, only being achieved at high enough deposition or annealing temperatures $\left[400{ }^{\circ} \mathrm{C}\right.$ for $\mathrm{FePd}$ (Ref. 5) and $600{ }^{\circ} \mathrm{C}$ for FePt (Ref. 6)], a fact that can be a drawback for the production of technological devices. In this scope alter-

\footnotetext{
${ }^{\text {a) }}$ Author to whom correspondence should be addressed; electronic mail: cesarcl@imm.cnm.csic.es
}

native methods used in recent years to obtain PMA in alloys grown at a lower temperature and with a smaller chemical ordering degree include the addition of third elements, ${ }^{7}$ artificial multilayering, ${ }^{8}$ ion irradiation, ${ }^{9}$ monoatomic layer control, ${ }^{10}$ compositional changes, ${ }^{11}$ and selection of buffer layer nature. ${ }^{12}$

This last method, a buffer layer between the substrate and the binary alloy, is generally used to increase the flatness of the initial growing surface and to decrease the existent lattice mismatch, and it has been demonstrated that the nature, ${ }^{5,10-17}$ thickness, ${ }^{5}$ and deposition temperature ${ }^{13}$ of the buffer layer affect strongly the ordering degree of the binary alloy. Amongst a large variety of materials, $\mathrm{V}$ has never been used as a buffer layer for the epitaxy of FePt and FePd alloys, in spite of the high flatness and crystalline quality of $\mathrm{V}(100)$ thin films grown on $\mathrm{MgO}(001)$ substrates $^{18}$ with interfacial roughness between 1 and $3 \AA$.

Therefore the motivation of this work is to study the structure, morphology, composition, magnetism, and magneto-optical (MO) response of FePd films sputter deposited at temperatures between room temperature (RT) and $700{ }^{\circ} \mathrm{C}$ on monocrystalline $\mathrm{V}(001)$ buffer layers grown on $\mathrm{MgO}(001)$ substrates. A PMA phase is obtained in FePd films grown at temperatures above $400{ }^{\circ} \mathrm{C}$ in spite of the fact that no chemical ordering is found. A strong $\mathrm{V}$ interdiffusion into the FePd layer and the formation of FeV alloy is accompanied by a two-dimensional to three-dimensional growth mode transition and an increase of the mosaic spread at these temperatures. Although in this case PMA is obtained at similar temperatures as in regular FePd alloys, in this approxima- 
tion FePd-FeV mixed systems can open alternative ways to obtain high anisotropy systems at lower temperatures and with lower chemical order requirements. The paper will be structured as follows: in Sec. II the experimental details are reported. In Sec. III the evolution of the different structural aspects, such as morphology, crystalline structure, and interface nature, as a function of the alloy deposition temperature will be described. Then Sec. IV will show the effects that these structural aspects have in different magnetic and magneto-optical properties, such as saturation magnetization, magnetic anisotropy, and magneto-optical activity. Finally, Sec. V will be devoted to presenting the main conclusions of the work.

\section{EXPERIMENT}

FePd films of $22 \mathrm{~nm}$ in thickness were obtained by triode sputtering codeposition from $\mathrm{Fe}$ and $\mathrm{Pd}$ targets at 4 $\times 10^{-4}$ mbar Ar pressure in an ultrahigh-vacuum chamber with a base pressure in the low $10^{-9}$ mbar range. The alloys were grown at different deposition temperatures, ranging from RT up to $700{ }^{\circ} \mathrm{C}$ at a deposition rate of $0.24 \AA / s$. Prior to FePd deposition, a $40 \AA$ buffer layer of $\mathrm{V}$ was deposited by sputtering at $400{ }^{\circ} \mathrm{C}$ on $\mathrm{MgO}(001)$ substrates at a deposition rate of $0.07 \AA$ /s, optimum conditions for the growth of high crystalline quality, and low surface roughness epitaxial V films. ${ }^{18}$ Subsequently to the FePd deposition, a $50 \AA$ thick protective capping layer of Pd was deposited at RT also by sputtering.

A multitechnique approach has been followed to study the fabricated structures, using atomic force microscopy (AFM), x-ray diffraction (XRD), and transmission electron microscopy (TEM) for the structural characterization, electron energy loss spectroscopy (EELS), and x-ray photoemission spectroscopy (XPS) for the in-depth resolved chemical composition, and superconducting quantum interference device (SQUID), magnetic force microscopy (MFM), magnetooptical Kerr effect (MOKE) loops, and Kerr spectroscopy for the magnetic and MO characterizations. AFM and MFM images were taken using a Nanotec ${ }^{\mathrm{TM}}$ microscope operating in a noncontact dynamic mode. For MFM, the tip scanned at a constant lift height above the sample (typically $50 \mathrm{~nm}$ ) and the phase shift, proportional to the force gradient, was measured. Probes with similar cantilevers (force constant $\sim 5 \mathrm{~N} / \mathrm{m}$ and resonant frequency $\sim 150 \mathrm{kHz}$ ) and pyramidal tips (cone angle below $20^{\circ}$ ) but different magnetic coatings were used. All of them were magnetized along the pyramid axis. XRD and X-ray reflectometry (XRR) experiments were performed in a four-circle diffractometer with $\mathrm{Cu} K \alpha(\lambda$ $=1.5418 \AA$ ) radiation, using Bragg-Brentano configuration and $1 / 4^{\circ}$ slits. TEM characterization was carried out in a Jeol 2010 field emission gun microscope operating at $200 \mathrm{kV}$. In these measurements, EELS was also used to detect variation in the composition of different areas of the samples. EELS spectra were taken within a range of electron energy loss rating from 325 to $745 \mathrm{eV}$, in order to examine $\mathrm{Pd} M_{4,5}$, V $L_{2,3}, \mathrm{O} K$, and Fe $L_{2,3}$ peaks. XPS depth profiling was carried out to investigate the concentration and chemical states of the elements in the layers; the samples were etched using $\mathrm{Ar}^{+}$ions with $1 \mathrm{keV}$ energy and the incidence angle of the impinging $\mathrm{Ar}^{+}$ions was $42^{\circ}$ with respect to the normal of the sample. The angle between the hemispherical analyzer (Specs, PHOIBOS 100) and the normal of the surface was kept at $30^{\circ}$. The quantitative analysis was performed by the evolution of the photoemission integrated intensities of $\mathrm{Fe}$ $2 p_{1 / 2}, \mathrm{Fe} 2 p_{3 / 2}, \mathrm{Pd} 3 d_{3 / 2}, \mathrm{Pd} 3 d_{5 / 2}, \mathrm{~V} 2 p_{1 / 2}$, and $\mathrm{V} 2 p_{3 / 2}$ transitions for each sample. Details on the determination of the $\mathrm{Ar}^{+}$etching rate can be found elsewhere. ${ }^{19}$

Magnetization measurements were carried out by using a rf-SQUID magnetometer from Quantum Design at RT in applied fields up to $20 \mathrm{kOe}$. The MO behavior of the samples was studied by means of transverse and polar MOKE loops. Transverse loops were measured with a $633 \mathrm{~nm}$ HeNe laser system at RT in $45^{\circ}$ incidence angle geometry; the change in reflectance was registered; in the case of polar Kerr loops, $530 \mathrm{~nm}$ light in normal incidence also at RT was used and the Kerr rotation angle was measured. Moreover, polar Kerr spectra were measured in a spectrometer similar to the one described in Ref. 20, in the spectral range from 1.4 to $4.3 \mathrm{eV}$.

\section{MORPHOLOGY AND STRUCTURE}

AFM images were taken in all samples to investigate the surface morphology dependence on the alloy deposition temperature. Since the surface roughness of a $40 \AA$ vanadium layer grown at $400{ }^{\circ} \mathrm{C}$ on $\mathrm{MgO}(001)$ is very low (with an interfacial roughness between 1 and $3 \AA),{ }^{18}$ we can then expect all the observed features in the as grown structures to be in principle primarily due to the Pd capped FePd alloy thin films. In Figs. 1(a)-1(f) we show the results for six of the samples, representative of the three different morphology regimes obtained as a function of deposition temperature. In the case of the low deposition temperature regime, we show AFM images for the samples grown at RT and $300{ }^{\circ} \mathrm{C}$ [Figs. 1(a) and 1(b)]. At RT can be observed a very flat surface and only small circular shaped features with an average in-plane diameter of $50 \mathrm{~nm}$ and $0.5 \mathrm{~nm}$ height, of the same order of what is observed in the bare $\mathrm{V}$ buffer layers; while in the $300{ }^{\circ} \mathrm{C}$ sample [Fig. 1(b)] these features are larger with an average in-plane diameter of $150 \mathrm{~nm}$ and $1.9 \mathrm{~nm}$ height. Morphological changes become evident as the FePd deposition temperature is increased, observing a transition from two-dimensional to three-dimensional growth mode at $400{ }^{\circ} \mathrm{C}$ [Fig. 1(c)], where the film morphology is not continuous anymore and the sample surface exhibits cracks with features of $200 \mathrm{~nm}$ in width in average lateral dimensions and $17 \mathrm{~nm}$ in depth (the nominal thickness of the film being $22 \mathrm{~nm}$ ). Above this temperature a clear three-dimensional growth mode is obtained, with the size of the nanoparticles continuously increasing with increasing deposition temperature. In Figs. 1(d)-1(f) we show AFM images for the samples grown at 500, 600, and $700{ }^{\circ} \mathrm{C}$, respectively, representative of this growth mode regime, observing nanoparticles with mean diameters ranging from 600 to $750 \mathrm{~nm}$ and average height from 40 to $50 \mathrm{~nm}$. In Fig. 1(g) AFM line scan profiles of samples grown at RT and 300, 400, 500, and 
(a) RT

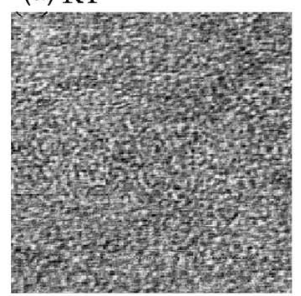

(c) $400^{\circ} \mathrm{C}$

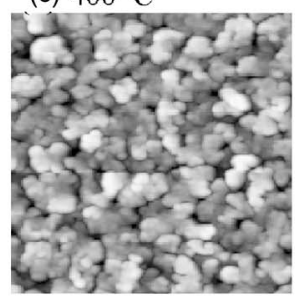

(e) $600^{\circ} \mathrm{C}$

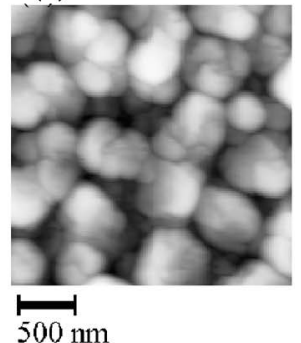

(g)

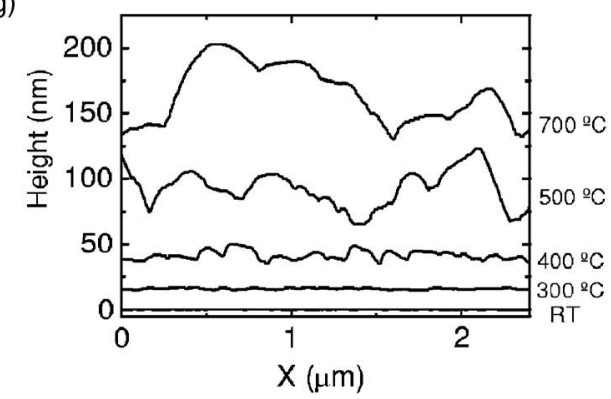

FIG. 1. AFM images corresponding to the samples grown at (a) RT and (b) 300, (c) 400, (d) 500, (e) 600, (f) and $700{ }^{\circ} \mathrm{C}$. (g) Profiles of the samples grown at RT and $300,400,500$, and $700{ }^{\circ} \mathrm{C}$. A two-dimensional to threedimensional growth mode transition is observed.

$700{ }^{\circ} \mathrm{C}$ are presented, where the lateral and vertical dimensions of the surface features of the samples can be easily compared.

Complementary structural information, now focused on the alloy crystalline structure, was obtained by XRD. Symmetric and asymmetric XRD scans as well as rocking curves around the main reflections were measured for all the samples. In Fig. 2 high angle symmetric scans are shown, where as reference bulk positions for $\operatorname{Pd}(200), \operatorname{FePd}(200)$, FePd(002), V(200), and FeV(200) peaks are indicated as dotted lines, extracted from Refs. 1, 21, and 22. The angular region around $2 \theta=24.4^{\circ}$, where the $\mathrm{FePd}(100)$ peak is generally observed in chemically ordered structures, is not shown since no (001) was detected in our case, regardless of the alloy deposition temperature. This implies that no chemically ordered $L 1_{0}$ phase is found in any sample. However, the strong evolution of the diffraction patterns as a function of deposition temperature indicates a temperature dependent structural modification at the atomic level in the layers. Re-

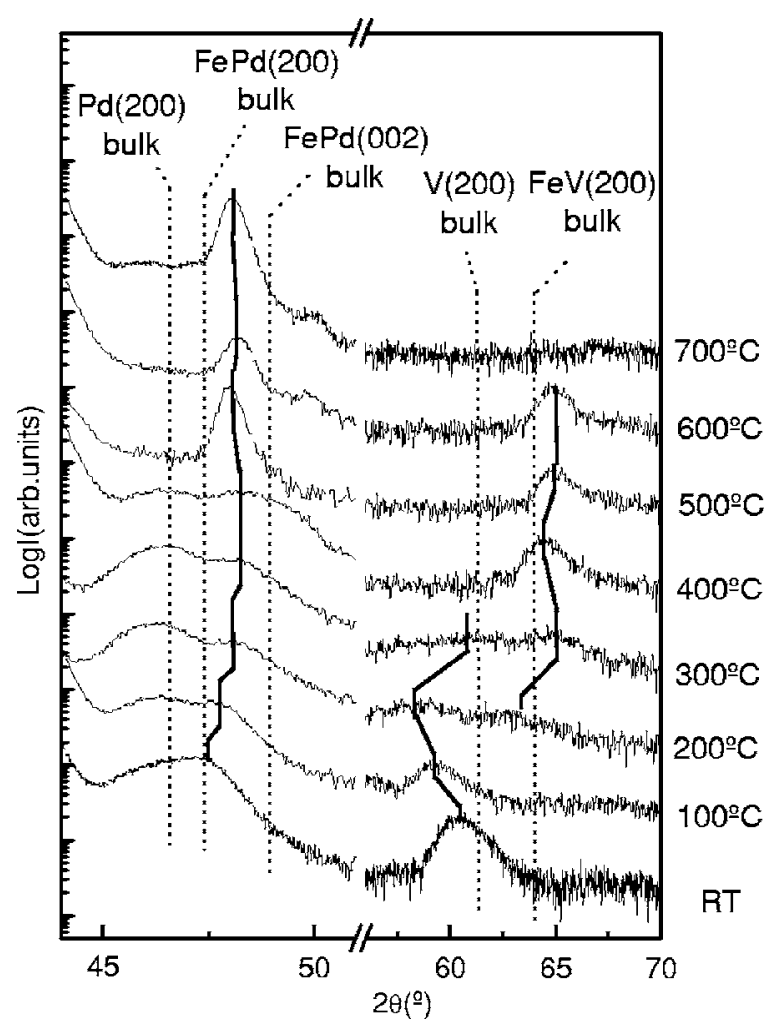

FIG. 2. Symmetric x-ray diffraction scans for the $\mathrm{Pd} / \mathrm{FePd} / \mathrm{V} / \mathrm{MgO}$ structures with different FePd deposition temperatures. The position of $\operatorname{Pd}(200)$, $\mathrm{FePd}(200), \mathrm{FePd}(002), \mathrm{V}(200)$, and $\mathrm{FeV}(200)$ bulk peaks is indicated.

garding the FePd layer, FePd(200) diffraction peaks appear in all the samples, corresponding to the chemically disordered phase of FePd; a smooth evolution of the position of this peak is observed, indicating a slight and continuous change in the alloy lattice parameter. Consistently with the absence of $\mathrm{FePd}(001)$ reflections, the FePd(002) ones, characteristic also of the ordered $L 1_{0} \mathrm{FePd}$ phase and that should appear around $2 \theta=49^{\circ},{ }^{13}$ were not observed independently of the deposition temperature. Only two weak peaks around $50^{\circ}$ appear for samples grown at 600 and $700{ }^{\circ} \mathrm{C}$ which could be due to a minoritary FePd ordered phase. Regarding the $\mathrm{V}$ buffer layer, the $\mathrm{V}(200)$ diffraction peak, whose bulk V position is $2 \theta=61.3^{\circ}$, is only detected at low deposition temperatures (RT and 100 and $200{ }^{\circ} \mathrm{C}$ ), shifting to smaller scattering angles, with a small signal near bulk position for $300{ }^{\circ} \mathrm{C}$, and disappearing in the sample grown at $400{ }^{\circ} \mathrm{C}$. On the other hand, an additional peak appears in the sample grown at $200{ }^{\circ} \mathrm{C}$ in positions around $2 \theta=65^{\circ}$, the intensity increasing with the growth temperature and being maximum in the $600{ }^{\circ} \mathrm{C}$ sample. It has been already observed in $\mathrm{Fe}-\mathrm{V}$ multilayers grown at high temperature ${ }^{23-25}$ the thermal induced diffusion of $\mathrm{V}$ into the $\mathrm{Fe}$ layer and the alloying of $\mathrm{Fe}$ and $\mathrm{V}$ forming a body centered cubic (bcc) or a tetragonal $\sigma$ phase depending on the stoichiometry and on the temperature. ${ }^{26}$ The lattice parameter for this tetragonal $\sigma$ phase is of $2.91 \AA{ }^{21}$ very close to the value extracted from the observed peaks. This is also very close to the $\mathrm{Fe}(200)$ reflection (Fe lattice parameter is $2.87 \AA$ ); therefore from these measurements it can be deduced that either the segregation of pure $\mathrm{Fe}$ regions or the formation of a $\mathrm{FeV}$ alloy 


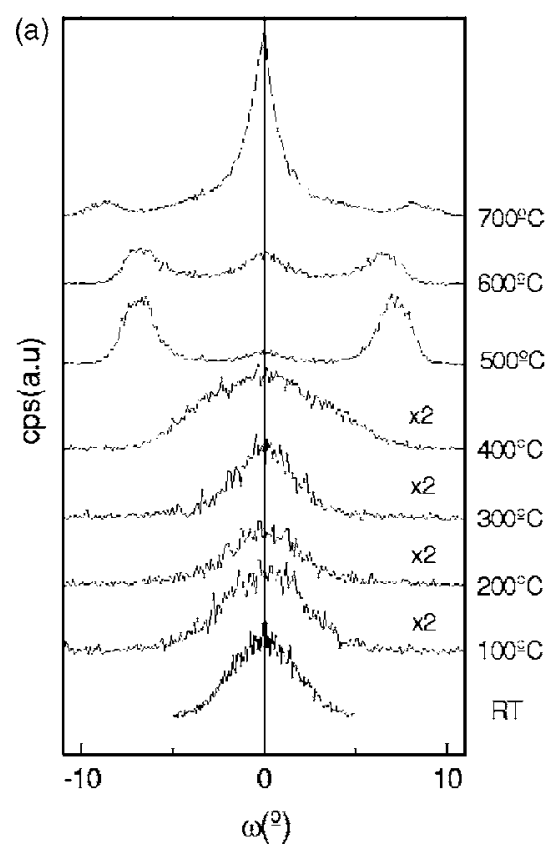

(b)
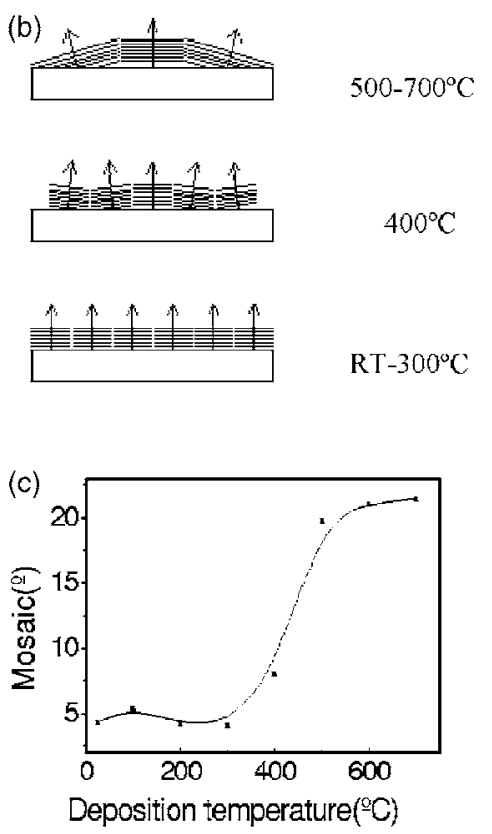

FIG. 3. (a) Rocking curves measured in the $\operatorname{FePd}(200)$ peaks, (b) sketch of the atomic plane evolution and (c) mosaic of the FePd films as a function of the deposition temperature. occurs. In the sample grown at $700{ }^{\circ} \mathrm{C}$ no trace of $\mathrm{V}$ or other compounds is found; this disappearance has been previously observed in $\mathrm{V}$ layers grown at high temperature on $\mathrm{MgO},{ }^{18}$ probably due to the $\mathrm{V}$ amorphization at these temperatures.

Additional structural information is presented in Fig. 3, where rocking curves measured around the $\operatorname{FePd}(200)$, $\mathrm{V}(200)$, and $\mathrm{FeV}(200)$ diffraction peaks are shown. The rocking curves corresponding to the $\mathrm{V}(200)$ diffraction peaks have an increasing width as the deposition temperature increases from RT to $300{ }^{\circ} \mathrm{C}$ (no V is found above $300{ }^{\circ} \mathrm{C}$ ), being extracted a mosaic spread ranging from $2^{\circ}$ to $10^{\circ}$. On the other hand rocking curves around the $\mathrm{FeV}(200)$ reflection exhibit a decreasing width with increasing temperature, ranging from $3^{\circ}$ in the $200{ }^{\circ} \mathrm{C}$ sample to $1.5^{\circ}$ at $600{ }^{\circ} \mathrm{C}$; no trace of $\mathrm{FeV}$ is found at $700{ }^{\circ} \mathrm{C}$. In the case of the $\mathrm{FePd}(200)$ peak [Fig. 3(a)], we observe distinctive regimes depending on the deposition temperature that clearly match those previously observed in AFM: from RT to $300{ }^{\circ} \mathrm{C}$, where a flat morphology was obtained, the FePd mosaic remains constant with values around $4^{\circ}$. At $400{ }^{\circ} \mathrm{C}$, where a transition from twodimensional to three-dimensional morphology happened, a sudden increase of a factor of 2 in the mosaicity up to $8^{\circ}$ is observed. Finally, above $400{ }^{\circ} \mathrm{C}$ the nature of the layer mosaic dispersion corresponds to that of a structure with a portion of crystallites aligned along the growth direction, as well as others misaligned with respect to it. In fact, for the sample grown at $500{ }^{\circ} \mathrm{C}$ the majority of crystallites are actually misaligned $7^{\circ}$ with respect to the growth direction, according to the relative peak areas observed in the corresponding rocking curve, with a very small portion of the alloy planes still normally aligned. For the $600{ }^{\circ} \mathrm{C}$ sample the portion of misaligned crystallites $\left(6.6^{\circ}\right.$ in this case) is reduced and that of the aligned ones increases, and finally for $700{ }^{\circ} \mathrm{C}$ most of the alloy particle crystalline planes (at this temperature the alloy is not continuous anymore) are aligned with the growth direction for this specific sample. In Fig. 3(b) a schematics of this interpretation about the mosaic structure evolution is de- picted, together with a quantitative evolution of the mosaic dispersion as a function of the alloy deposition temperature in Fig. 3(c). There, the assigned values for the mosaic spread for 500-700 ${ }^{\circ} \mathrm{C}$ samples correspond to the separation of the measured peaks for the crystallites misaligned with respect to the growth direction.

The epitaxial relations between the substrate, buffer, and alloy layers were determined by measuring the asymmetric $\mathrm{FePd}(220), \mathrm{V}(110)$, and $\mathrm{MgO}(110)$ reflections together with the $\mathrm{V}(200)$ and $\mathrm{FePd}(200)$ symmetric reflections, concluding the epitaxial relation: $[010](001) \mathrm{FePd}\|[-110](001) \mathrm{V}\|[010]$ $\times(001) \mathrm{MgO}$, i.e., a $45^{\circ}$ in-plane rotation of the $\mathrm{V}[100] \mathrm{di}-$ rection with respect to the $\mathrm{MgO}$ and FePd ones. The out-ofplane (c) and in-plane (a) lattice parameters of the FePd, $\mathrm{FeV}$, and $\mathrm{V}$ layers were also extracted from the position of the mentioned symmetric and asymmetric reflections, the corresponding results being presented in Fig. 4. The bulk lattice parameters are marked with a dashed line. A very small tetragonal distortion is observed in $\mathrm{FePd}$ at moderated deposition temperatures $(c / a=0.99)$, much smaller than it would be expected for an ordered phase $(c / a=0.97){ }^{22} \mathrm{~A}$ tetragonal distortion is also observed in the FeV alloy phase, with a $c / a$ ratio of 0.86 .

In order to confirm the $\mathrm{V}$ diffusion in the FePd layer and to investigate the morphology and composition of the different layers, a combined TEM and EELS study was performed. This allows a simultaneous structural and chemical characterization with high spatial resolution. At low FePd deposition temperatures $\left(100,200\right.$, and $\left.300{ }^{\circ} \mathrm{C}\right)$ very flat and crystalline alloy layers are observed, the epitaxial relationship with the substrate measured with XRD being confirmed. No interdiffusion of $\mathrm{V}$ or Pd appears in the FePd layers grown at low temperature as the EELS spectra demonstrate. The $\mathrm{V}(200)$ peak shift observed in the XRD between RT and $200{ }^{\circ} \mathrm{C}$ might be then due to epitaxial strain or very small interdiffusion, below the EELS detection limits. Figure 5(a) shows a TEM micrograph of the sample grown at $100{ }^{\circ} \mathrm{C}$, 


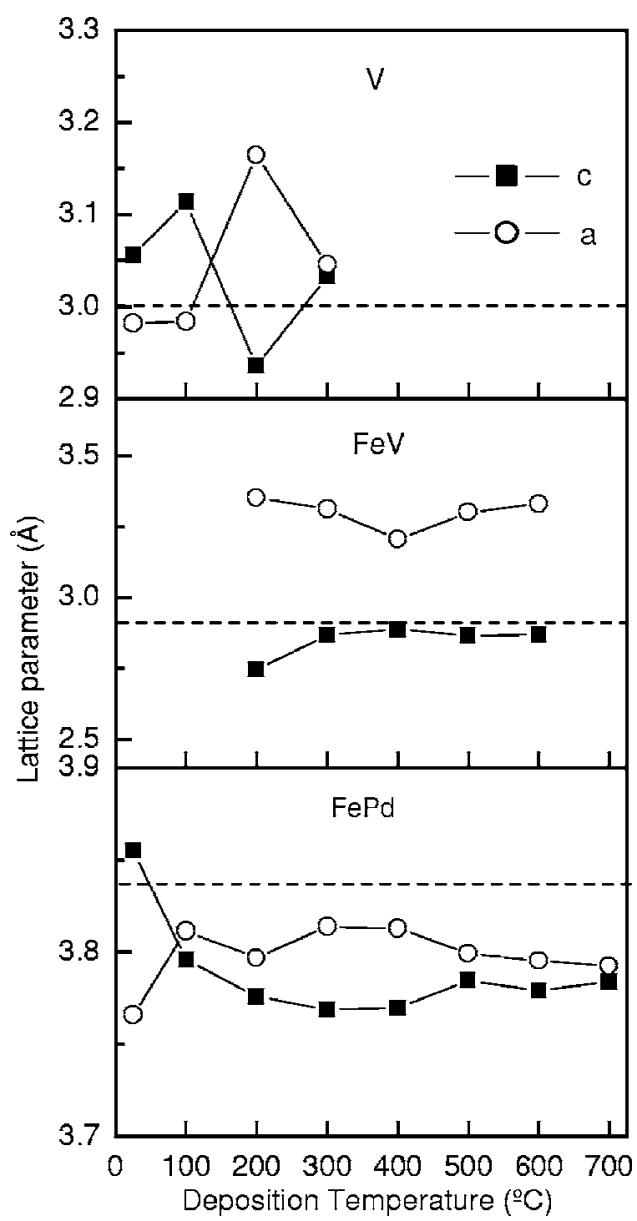

FIG. 4. Determined in-plane (a) and out-of-plane (c) lattice parameters of V, $\mathrm{FeV}$, and $\mathrm{FePd}$ as a function of the deposition temperature. The bulk values are indicated in each case by a dashed line.

with a very well defined layered structure and clear interfaces: $\mathrm{MgO}, \mathrm{V}$, and FePd layers can be easily distinguished. Different EELS spectra were measured in the circled areas to investigate possible interdiffusion and the presence of $\mathrm{V}$ in the FePd layer. In Fig. 5(b) the spectra for the $100{ }^{\circ} \mathrm{C}$ sample measured in the marked areas are shown; the different peaks corresponding to the $\mathrm{Pd} M_{4,5}, \mathrm{~V} L_{2,3}, \mathrm{O} K$, and $\mathrm{Fe} L_{2,3}$ transitions were investigated. However, no trace of the $\mathrm{V} L_{2,3}$ peak appears in the FePd layer. In the sample grown at $400{ }^{\circ} \mathrm{C}$, where a $\mathrm{FeV}$ alloy diffraction peak is already observed, the layered structure is not as well defined as for lower deposition temperatures [Fig. 5(c)]. Again, EELS spectra were taken in the circled regions [Fig. 5(d)] and this time $\mathrm{V}$ is found in the FePd layer, with a $\mathrm{V}$ content which is higher near the $\mathrm{FePd}-\mathrm{V}$ interface, and that gradually reduces as we move farther away from it. This interdiffusion is also observed in the samples grown at higher temperature with increasing intensity. In the $700{ }^{\circ} \mathrm{C}$ sample there is no possibility to distinguish separate $\mathrm{V}$ and FePd layers [Fig. 5(e)] and the $\mathrm{V}$ concentration is found to be constant in all the FePd layers according to the relative $\mathrm{V} L_{2,3}$ peak areas observed [Fig. 5(f)].

Complementary depth profiling XPS measurements were performed on samples grown at RT and high temperature $\left(600^{\circ} \mathrm{C}\right)$. The resulting concentrations of the different ele- ments of interest ( $\mathrm{Fe}, \mathrm{Pd}, \mathrm{V}, \mathrm{Mg}$, and $\mathrm{O}$ ) as a function of thickness and for the two samples are presented in Fig. 6. As can be seen, for the sample grown at RT [Fig. 6(a)] the concentration of V exhibits a Gaussian distribution quenched in between the FePd film and the $\mathrm{MgO}$ substrate, indicating the presence of sharp interfaces and consequently the absence of $\mathrm{V}$ diffusion into the FePd thin film and in agreement with the conclusions extracted from the EELS measurements in the alloy grown at $100{ }^{\circ} \mathrm{C}$. Note that the observation of sharp interfaces also indicates that no implantation phenomena are induced with $\mathrm{Ar}^{+}$ion with $1 \mathrm{keV}$ kinetic energy. On the other hand, it can be clearly seen that for the sample grown at $600{ }^{\circ} \mathrm{C}$ [Fig. 6(b)] $\mathrm{V}$ is distributed in the whole FePd film and capping layer and that no sharp interfaces are observed. Such distribution of the V concentration as a function of thickness clearly indicates that, in contrast to the sample grown at RT, there is a strong diffusion of $\mathrm{V}$ into the FePd film. No clear changes in the chemical states of the V, $\mathrm{Pd}$, and Fe elements could be observed on the core level spectra, probably due to the low concentration (always less than 5\%) of the $\mathrm{V}$ and $\mathrm{FeV}$ alloys in the samples. As can be observed, the concentration ratio between $\mathrm{Fe}$ and $\mathrm{Pd}$ is close to 0.5 and almost constant in all the FePd layers and for both samples indicating the growth of a homogeneous and stoichiometric FePd compound.

\section{MAGNETIC AND MAGNETO-OPTICAL PROPERTIES}

SQUID magnetometry has been used to obtain the saturation magnetization $\left(M_{s}\right)$ of the samples. A value close to that of the stoichiometric phase at RT $\left(1280 \mathrm{emu} / \mathrm{cm}^{3}\right)($ Ref. 14 ) is found in the samples grown at lower temperatures (from RT to $300{ }^{\circ} \mathrm{C}$ ), while a strong reduction is observed in the samples grown at higher temperature $(400,500,600$, and $\left.700{ }^{\circ} \mathrm{C}\right)$ [Fig. 7(a)]. This change appears in the sample grown at $400{ }^{\circ} \mathrm{C}$, in which the onset of $\mathrm{V}$ diffusion was observed by EELS; therefore the diffusion of $\mathrm{V}$ into the FePd layer and the appearance of $\mathrm{FeV}$ alloy with a lower average magnetization moment can explain the decrease of the saturation magnetization. It has been demonstrated that in $\mathrm{Fe}-\mathrm{V}$ interfaces $\mathrm{V}$ couples antiferromagnetically with $\mathrm{Fe}$, giving rise to a induced magnetization in $\mathrm{V}$ (magnetic moment per $\mathrm{V}$ atom ranging from $0.3 \mu_{B}$ to $1.5 \mu_{B}$ ), and a $20 \%$ decrease in the magnetic moment of $\mathrm{Fe}$ interface atoms. ${ }^{27,28}$ This reduction has been observed as well in $\mathrm{FeV}$ alloys, measuring a magnetization of $0.99 \mu_{B} /$ at. for $\mathrm{Fe}$ (55\% reduction) in equiatomic alloys ${ }^{29}$ or even the total extinction for high concentrations of V. On the other hand in FePd the coupling is ferromagnetic and it has been calculated a magnetic moment of $3.04 \mu_{B}$ /at. for Fe exceeding the Fe value $\left(2.2 \mu_{B} /\right.$ at. $)$ and of $0.38 \mu_{B}$ /at. for $\mathrm{Pd}^{30}$

Hysteresis loops for out-of-plane and in-plane fields have been measured by polar and transverse MOKEs; in Fig. 7(b) a complete characterization is shown: polar loops on the left column and the corresponding transverse loops on the right. In order to understand the magnetic behavior of the system we must keep in mind that two factors are competing: on one hand, the change in morphology from continuous 
$100^{\circ} \mathrm{C}$

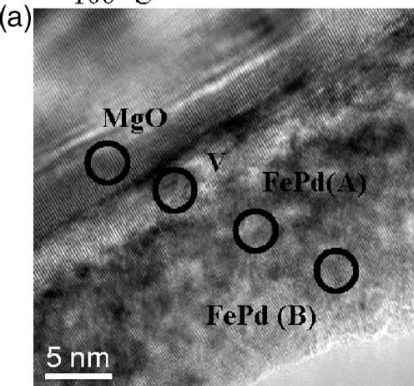

$400^{\circ} \mathrm{C}$

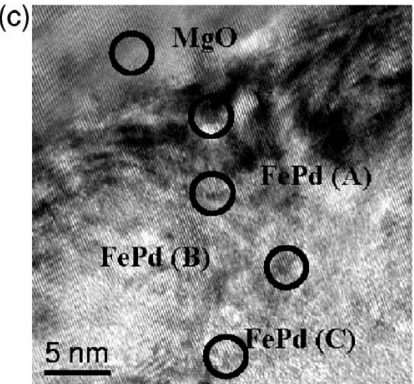

$700{ }^{\circ} \mathrm{C}$

(e)

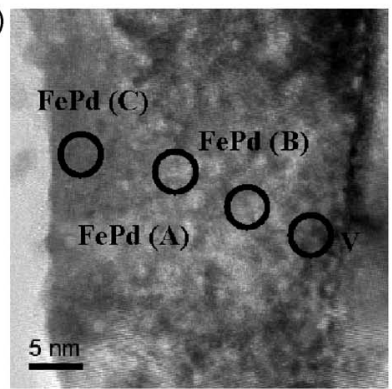

(b)
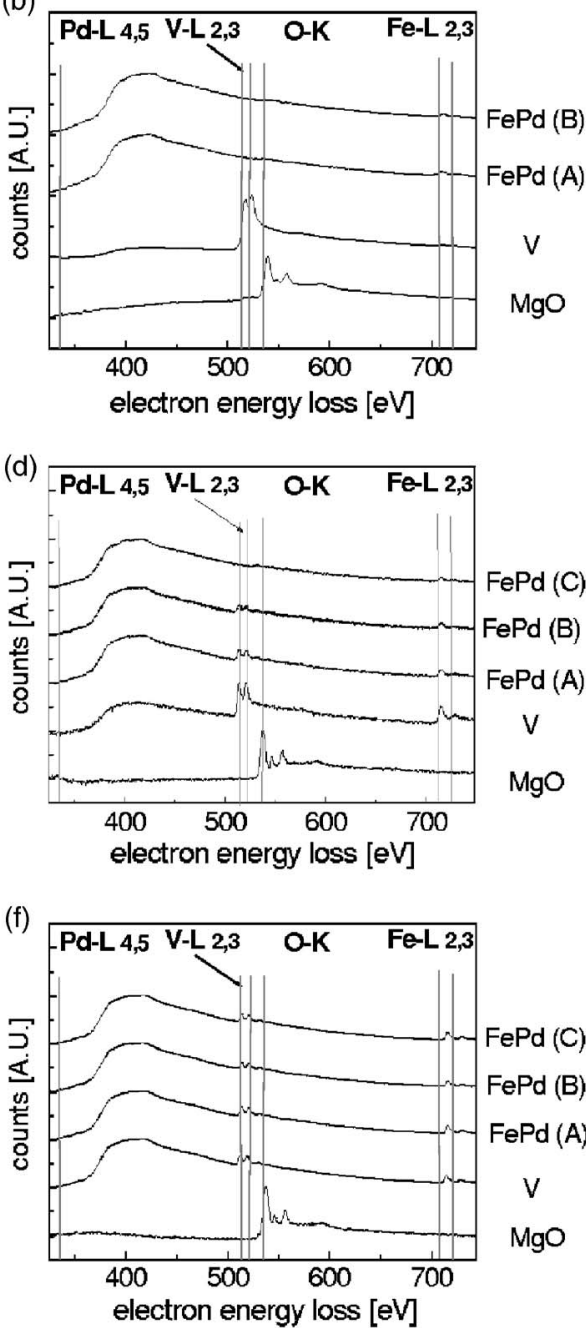

FIG. 5. (a), (c), and (e) TEM micrograph of the samples grown at 100,400 , and $700{ }^{\circ} \mathrm{C}$, respectively. (b), (d), and (f) EELS spectra for the mentioned samples measured in the points marked in the images shown beside. The peaks Pd $M_{4,5}, \mathrm{~V} L_{2,3}, \mathrm{O} K$, and Fe $L_{2,3}$ are studied. Note the absence of $\mathrm{V}$ in the FePd layer in the $100{ }^{\circ} \mathrm{C}$ sample, how $\mathrm{V}$ appears in different points of the $\mathrm{FePd}$ layer in the $400{ }^{\circ} \mathrm{C}$ sample, and the constant concentration of $\mathrm{V}$ along the $\mathrm{FePd}$ layer in the $700{ }^{\circ} \mathrm{C}$ sample. films to three-dimensional islands as deposition temperature increases; and on the other hand, the $\mathrm{FeV}$ alloy formation between 400 and $700{ }^{\circ} \mathrm{C}$ as a consequence of the $\mathrm{V}$ interdiffusion. The samples grown at RT and 100 and $200{ }^{\circ} \mathrm{C}$ show a typical behavior of continuous thin films with in-plane magnetic anisotropy; they have a small in-plane coercive field and saturation field, and high out-of-plane saturation field. $^{31}$ They also exhibit a fourfold anisotropy, with easy [100] directions and hard [110] ones [shown in the transverse loops in Fig. 7(b)]. The sample grown at $300{ }^{\circ} \mathrm{C}$ is similar but the fourfold anisotropy has disappeared, probably due to the appearance of cracks in the layer that produces an additional configurational anisotropy which smears out the crystalline counterpart, as previously evidenced in $\mathrm{Fe} / \mathrm{MgO}(001) .{ }^{31}$ Again, the sample grown at $400{ }^{\circ} \mathrm{C}$ separates two different behavior regions in the magnetic response as it did in the structure and saturation magnetization. The in-plane coercive field increases from 6 to $150 \mathrm{Oe}$, due mainly to an increase in the nanoparticle height at that temperature (as observed by AFM and TEM) which leads to a smaller physical connection and therefore a weaker effective magnetic exchange between the nanoparticles; but also to the high dispersion of crystalline plane orientation (mosaic spread) observed in this sample. The increase of the out-of- plane coercive field and the big reduction of the out-of-plane saturation field point to the presence of perpendicular anisotropy, in spite of the absence of chemical order at this deposition temperature. Also in SQUID measurements where a higher field is applied and the sample gets saturated, an enormous increase of the in-plane saturation field $(6 \mathrm{kOe})$ is observed in this sample, confirming the presence of a phase with perpendicular anisotropy. The samples grown at 500, 600 , and $700{ }^{\circ} \mathrm{C}$ show a similar response as the one grown at $400{ }^{\circ} \mathrm{C}$, with decreasing in-plane and out-of-plane coercivities. Also a decrease of the in-plane and an increase of the out-of-plane saturation fields are observed, indicative of a PMA phase. It is worth noticing that the PMA cannot be ascribed to the three-dimensional (3D) growth, since the nanoparticles exhibit a disklike shape with a maximum height/diameter ratio around 0.1 that is unlikely to promote perpendicular magnetization.

This clear correlation between reduced $M_{s}$ (induced by a $\mathrm{FeV}$ alloy formation) and PMA, simultaneously observed in all the samples grown between 400 and $700{ }^{\circ} \mathrm{C}$, indicates that the presence of the $\mathrm{FeV}$ alloy is responsible for the PMA. The magnetic anisotropy constant $\left(K_{u}\right)$ calculated for the samples exhibiting PMA from the SQUID hysteresis loops measured along the in-plane direction reaches values 

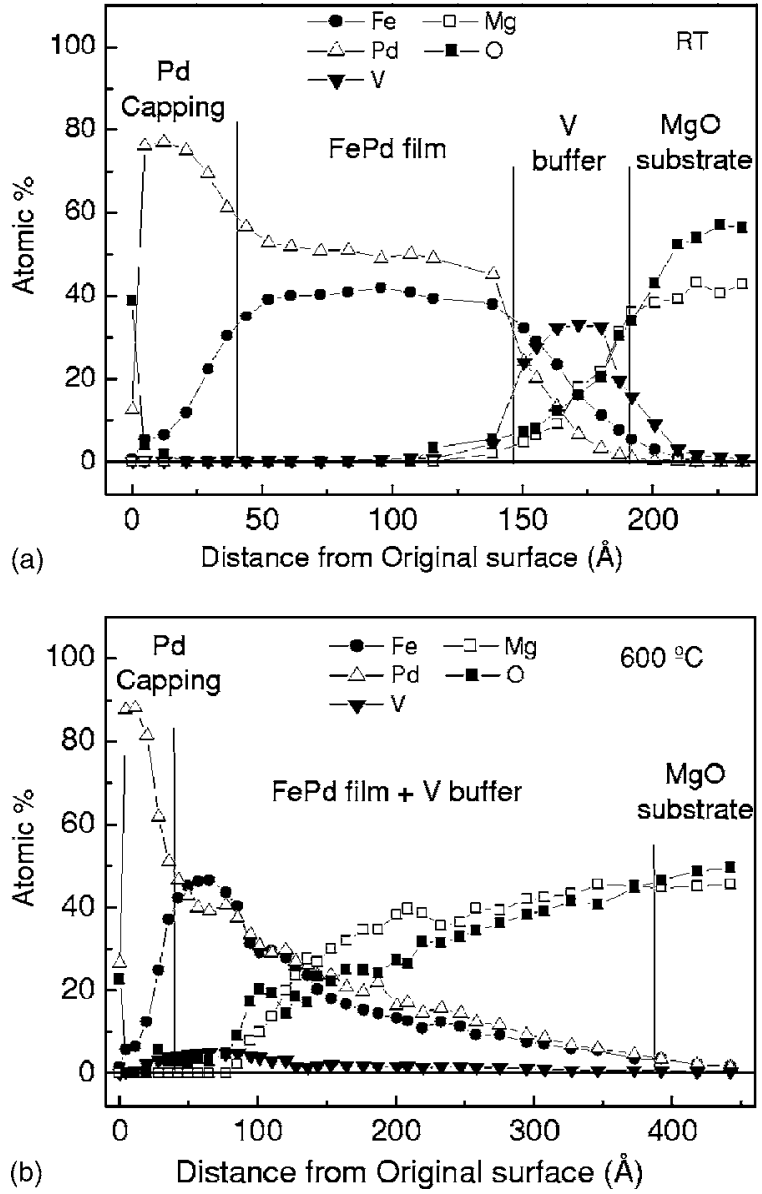

FIG. 6. Evolution of $\mathrm{Pd}, \mathrm{Fe}, \mathrm{V}, \mathrm{Mg}$, and $\mathrm{O}$ concentrations vs the distance from the surface, as detected by XPS for the samples grown at RT (a) and $600{ }^{\circ} \mathrm{C}(\mathrm{b})$.

of $5.2 \times 10^{4} \mathrm{~J} / \mathrm{m}^{3}$ for the $400{ }^{\circ} \mathrm{C}$ sample and $2.34 \times 10^{4}$ and $1.25 \times 10^{4} \mathrm{~J} / \mathrm{m}^{3}$ for the 500 and $700{ }^{\circ} \mathrm{C}$ samples, respectively. These values are quite below the one found in stoichiometric $L 1_{0}$ FePd ordered alloy $\left(9 \times 10^{5} \mathrm{~J} / \mathrm{m}^{3}\right)$ but are quite high for a chemically disordered alloy.

MFM measurements confirm these findings. Figure 8 shows MFM images of three different samples at zero magnetic field. The images obtained with samples grown at high temperature exhibit alternate dark and bright regions, which is the distinctive feature of samples with perpendicular anisotropy: $:^{32,33}$ these alternate regions correspond to up and down orientations of the magnetization. However, the sample grown at RT exhibits narrow lines that can be ascribed to magnetic domain walls ${ }^{34}$ meaning that elsewhere the magnetization lies mainly in plane of the film. It is worth noticing that low moment tips were needed in this MFM experiment to prevent the tip from dragging the domain walls.

To explore possible changes in electronic structure related with the $\mathrm{FeV}$ alloy formation, the $\mathrm{MO}$ spectral response of all the samples was investigated with polar Kerr spectroscopy in the photon energy range from 1.4 to $4.3 \mathrm{eV}$. In Fig. 9 the evolution of the Kerr rotation spectra as a function of deposition temperature is shown. While the samples grown at low temperature (RT and 100, 200, and $300{ }^{\circ} \mathrm{C}$ ) show almost identical spectra, a strong change is observed in the sample grown at $400{ }^{\circ} \mathrm{C}$, with an overall reduction of the
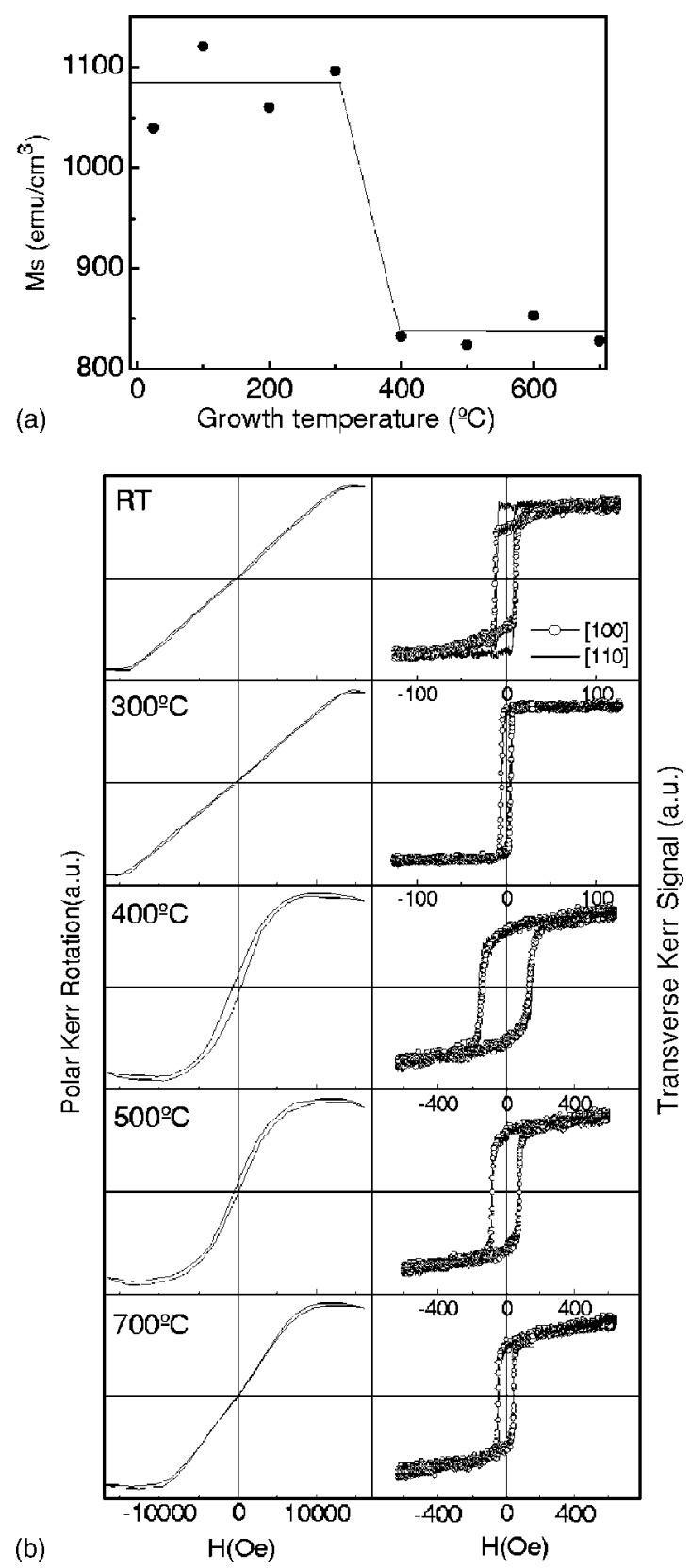

FIG. 7. (a) Saturation magnetization of the samples as a function of the deposition temperature measured with SQUID; the line is just a guide for the eyes. (b) Evolution of the polar (left) and transverse (right) Kerr loops with temperature. For the transverse loops, two curves are shown, one obtained for the [100] direction and the other for the [110] direction. Please note the different scales in the transverse loops.

Kerr rotation intensity and an even stronger reduction of the intensity of the $4 \mathrm{eV}$ Kerr rotation peak in the sample grown at $700{ }^{\circ} \mathrm{C}$. In FePd alloys this peak at $4 \mathrm{eV}$ is related to the polarization of Pd induced by $\mathrm{Fe},{ }^{35}$ the reduction of the intensity of this peak as we increase the growth temperature probably due to the presence of a $\mathrm{FeV}$ alloy. The $\mathrm{V}$ atoms will reduce the number of $\mathrm{Pd}$ atoms in contact with $\mathrm{Fe}$ and therefore their polarization, which will produce a reduction of the intensity of the Kerr rotation. The amount of $\mathrm{FeV}$ increases as deposition temperature increases due to the total diffusion of $\mathrm{V}$ in the FePd layers, as observed by EELS and XPS. 

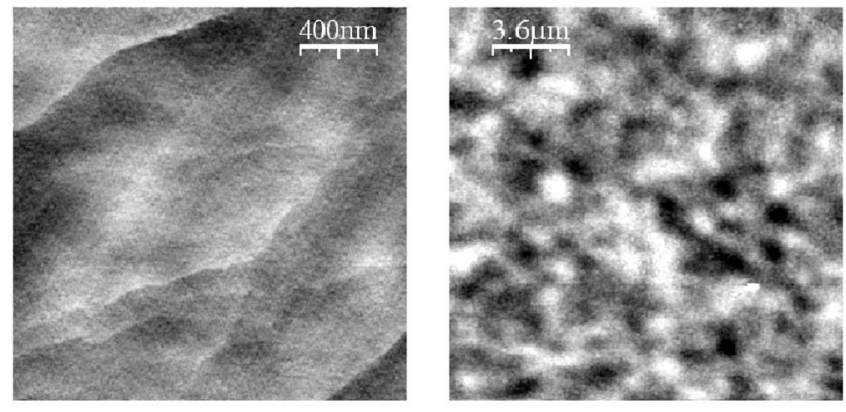

RT

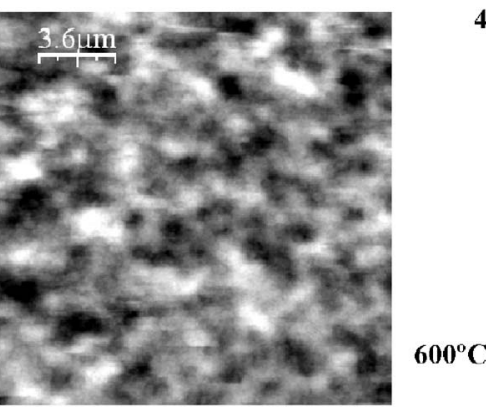

$400^{\circ} \mathrm{C}$

$600^{\circ} \mathrm{C}$

FIG. 8. MFM images of different samples. Sizes of $2 \times 2 \mu \mathrm{m}^{2}$ for the RT sample and $18 \times 18 \mu \mathrm{m}^{2}$ for the 400 and $600{ }^{\circ} \mathrm{C}$ samples.

\section{CONCLUSIONS}

We have studied the structure, morphology, composition, magnetism, and MO response of FePd films sputter deposited at different temperatures (from RT to $700{ }^{\circ} \mathrm{C}$ ) on a monocrystalline $\mathrm{V}(001)$ buffer layer. Above $400{ }^{\circ} \mathrm{C}$ a strong $\mathrm{V}$ interdiffusion into the FePd layer and the formation of $\mathrm{FeV}$ alloy is accompanied by a two-dimensional to threedimensional growth mode transition and an increase of the mosaic spread at these temperatures. A PMA phase is found in $\mathrm{FeV}-\mathrm{FePd}$ films grown at temperatures above $400{ }^{\circ} \mathrm{C}$ in spite of the absence of long range chemical ordering, usually

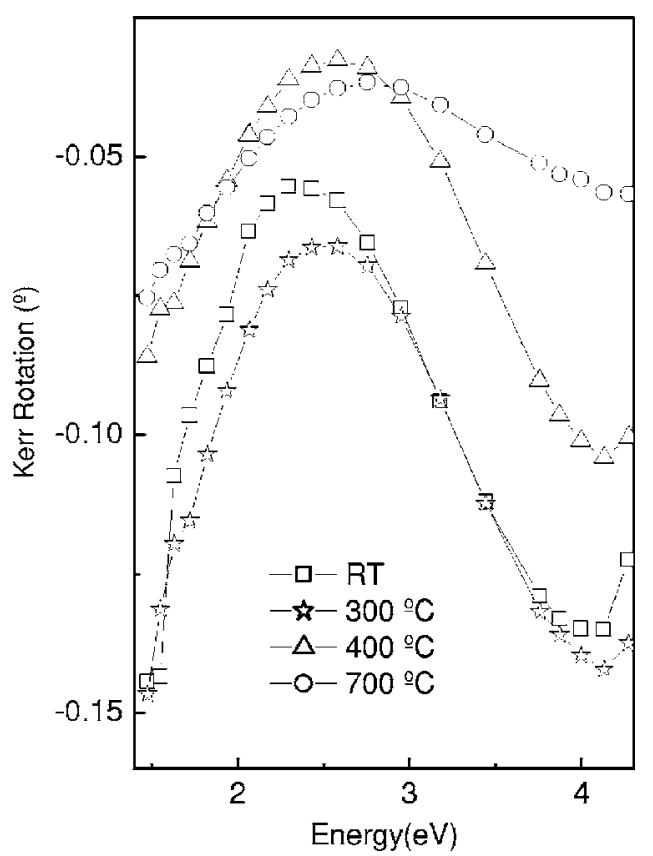

FIG. 9. Polar Kerr rotation spectra of the samples grown at different temperatures. observed in FePd systems at high deposition temperature. We attribute the observed PMA to the $\mathrm{V}$ interdiffusion into the FePd layer and the formation of a FeV alloy. Although in this case PMA is obtained at similar temperatures as in regular FePd alloys, this approximation, i.e., FePd-FeV mixed systems, can open alternative ways to obtain high anisotropy systems at lower temperatures and with lower chemical order.

\section{ACKNOWLEDGMENTS}

This work was partially financed by the Spanish Commission of Science and Technology and Comunidad de Madrid. One of the authors (C.C.) acknowledges the Ministerio de Educación y Ciencia and FPI program for financial support. Two of the authors (J.M.G.-M. and Y.H.) acknowledge the Consejo Superior de Investigaciones Científicas (CSIC) and Ramón y Cajal program for financial support. The authors thank Dr. Elisa Román for the use of the XPS equipment and fruitful discussions.

${ }^{1}$ A. Cebollada, R. F. C. Farrow, and M. F. Toney, in Magnetic Nanostructures, edited by H. S. Nalwa (American Scientific Publishers, Los Angeles, 2002).

${ }^{2}$ D. Weller and A. Moser, IEEE Trans. Magn. 35, 4423 (1999).

${ }^{3}$ S. Sun, C. B. Murray, D. Weller, L. Folks, and A. Moser, Science 287, 1989 (2000).

${ }^{4}$ S. Sun, E. E. Fullerton, D. Weller, and C. B. Murray, IEEE Trans. Magn. 37, 1239 (2001)

${ }^{5}$ P. Caro, A. Cebollada, F. Briones, and M. F. Toney, J. Cryst. Growth 187, 426 (1998).

${ }^{6}$ T. Shima, K. Takanashi, Y. K. Takahashi, K. Hono, G. Q. Li, and S. Ishio, J. Magn. Magn. Mater. 266, 171 (2003).

${ }^{7}$ T. Maeda, T. Kai, A. Kikitsu, T. Nagase, and J. Akiyama, Appl. Phys. Lett. 80, 2147 (2002).

${ }^{8}$ Y. Endo, N. Kikuchi, O. Kitakami, and Y. Shimada, J. Appl. Phys. 89, 7065 (2001).

${ }^{9}$ D. Ravelosona, C. Chappert, H. Bernas, D. Halley, Y. Samson, and A. Marty, J. Appl. Phys. 91, 8082 (2002).

${ }^{10}$ T. Shima, T. Moriguchi, S. Mitani, and K. Takanashi, Appl. Phys. Lett. 80, 288 (2002).

${ }^{11}$ T. Seki, T. Shima, K. Takanashi, Y. Takahashi, and E. Matsubara, Appl. Phys. Lett. 82, 2461 (2003).

${ }^{12}$ Y. N. Hsu, S. Jeong, D. E. Laughlin, and D. N. Lambeth, J. Appl. Phys. 89, 7068 (2001)

${ }^{13}$ P. Caro, A. Cebollada, D. Ravelosona, J. Tamayo, R. García, and F. Briones, Acta Mater. 46, 2299 (1998).

${ }^{14}$ P. Caro, A. Cebollada, D. Ravelosona, F. Briones, D. García, M. Vázquez, and A. Hernando, J. Appl. Phys. 81, 5050 (1997).

${ }^{15}$ M. F. Toney, W. Y. Lee, J. A. Hedstrom, and A. Kellock, J. Appl. Phys. 93, 9902 (2003).

${ }^{16}$ D. Halley, B. Gilles, P. Bayle-Guillemaud, R. Arenal, A. Marty, G. Patrat, and Y. Samson, Phys. Rev. B 70, 174437 (2004).

${ }^{17}$ Y. K. Takahashi, K. Hono, T. Shima, and K. Takanashi, J. Magn. Magn. Mater. 267, 248 (2003).

${ }^{18}$ Y. Huttel, E. Navarro, and A. Cebollada, J. Cryst. Growth 273, 474 (2005).

${ }^{19}$ M. S. Martín-González, Y. Huttel, A. Cebollada, G. Armelles, and F. Briones, Surf. Sci. 571, 63 (2004).

${ }^{20}$ W. S. Kim, M. Aderholz, and W. Kleemann, Meas. Sci. Technol. 4, 1275 (1993).

${ }^{21}$ M. Talanana, M. Benakki, F. Amalou, S. Bouarab, and C. Demangeat, Eur. Phys. J. B 22, 497 (2001).

${ }^{22}$ W. Pearson, Handbook of Lattice Spacings and Structures of Metals (Pergamon, New York, 1958).

${ }^{23}$ A. Scherz, P. Poulopoulos, R. Nünthel, J. Lindner, H. Wende, F. Wilhelm, and K. Baberschke, Phys. Rev. B 68, 140401 (2003).

${ }^{24} \mathrm{G}$. Andersson, E. Nordström, and R. Wäppling, Europhys. Lett. 60, 731 (2002).

${ }^{25}$ J. F. M. Borges, J. B. M. da Cunha, and M. I. da Costa, Jr., J. Phys.: 
Condens. Matter 15, 1 (2003).

${ }^{26}$ J. M. Sanchez, M. C. Cadeville, V. Pierron-Bohnes, and G. Inden, Phys. Rev. B 54, 8958 (1996).

${ }^{27}$ J. Izquierdo, A. Vega, O. Elmouhssine, H. Dreyssé, and C. Demangeat, Phys. Rev. B 59, 14510 (1999).

${ }^{28}$ H. Fritzsche, Y. T. Liu, J. Hauschild, and H. Maletta, Phys. Rev. B 70, 214406 (2004).

${ }^{29}$ J. C. Krause, J. Schaf, and M. I. da Costa, Jr., Phys. Rev. B 61, 6196 (2000).

${ }^{30}$ D. García, R. Casero, M. Vázquez, and A. Hernando, Phys. Rev. B 63, 104421 (2001).
${ }^{31}$ F. Cebollada, A. Hernando-Mañeru, A. Hernando, C. Martínez-Boubeta, A. Cebollada, and J. M. Gonzalez, Phys. Rev. B 66, 174410 (2002).

${ }^{32}$ V. Gehanno, A. Marty, B. Gilles, and Y. Samson, Phys. Rev. B 55, 12552 (1997).

${ }^{33}$ A. Asenjo, J. M. García, D. García, A. Hernando, M. Vázquez, P. A. Caro, D. Ravelosona, A. Cebollada, and F. Briones, J. Magn. Magn. Mater. 196-197, 23 (1999).

${ }^{34}$ A. Wadas, M. Dreyer, M. Kleiber, and R. Wiesendanger, Appl. Phys. A: Mater. Sci. Process. 66, 465 (1998).

${ }^{35} \mathrm{G}$. Armelles, D. Weller, B. Rellinghaus, P. Caro, A. Cebollada, and F. Briones, J. Appl. Phys. 82, 4449 (1997). 